\section{Pre-lens tear film evaluation scale: a tool for analyzing the tear film-lens interaction with respect to the material used}

\author{
Antonio Papagni, Rossella Fonte, \\ Luca Benzoni \\ Department of Materials Sciences, \\ University of Milano-Bicocca, Milano, \\ Italy
}

\begin{abstract}
In clinical practice, to avoid or reduce the failure rate among users of contact lenses, it is necessary to obtain a detailed patient case history, information concerning symptoms, and a complete eye examination. In addition, application of an auxiliary photographic reference scale might increase the chances of improving initial success or, for existing contact lens wearers, provide insight into the extent or severity of symptoms. Patient symptoms are often directly related to contact lens failure, which in turn is related to the quality of the pre-corneal tear film as well as the wettability of the contact lens surface. We describe the relationship between the quality of the tear film, with particular attention to pre-contact lens tear film, variability in contact tolerance, and the material of which the contact lens is made.
\end{abstract}

\section{Introduction}

The causes of contact lens (CL) dropout are frequently misunderstood and underestimated. Recent studies ${ }^{1}$ have reported mean dropout rates were $15.9 \%$ in the United States, $17.0 \%$ in the Americas (including the US), $31.0 \%$ in the Asian-Pacific Rim, and 30.4\% in Europe, the Middle East and Africa. These rates were all statistically significantly higher than the historically reported rate of $10 \%$. The most common reason for CL dropout included discomfort (41.9-52.9\%), which is strongly related to the tear film conditions and the type of materials used.

As confirmed in the literature, there are no obvious correlations between materials and tolerability of the hydrogel lenses, and even recent studies examine measurement of lens performance and dehydration in vitro. ${ }^{2-6}$ This study aims to identify a correlation between the appearance of the surface of the lens and its subjective tolerability. Furthermore, by using a photographic scale we propose a more objective clinical method that can help the optometrist in the choice of the proper material as a function of the tear or the dehydration of the surface observed after a blink. This could lead to a reduced incidence of dropouts because symptoms such as dryness and discomfort are very common in hydrogel CL wearers. ${ }^{7}$

\section{Materials and Methods}

\section{Subjects}

This clinical observational trial study involved 84 healthy eyes of 42 subjects: 12 males, 24 females, mean age 25.1 years. Refractive condition was up to 8.00 diopters (D) of myopia and 4.00 (D) of hyperopia, and astigmatism was less than $0.75 \mathrm{D}$. Study subjects were divided into two groups of 21 individuals each: Group 1 consisted of subjects who regularly wear CL (5 males, 16 are females); Group 2 was made up of occasional wearers or those wearing daily disposable lenses (13 males, 8 females).

The main aim of this study was to find a relationship between the quality of the precorneal tear film using a variety of lens materials. In order to achieve this, subjects were divided into different categories, according to the make-up of the pre-corneal tear film quality as determined by analysis using the slit lamp biomicroscope in combination with Tearscope Plus ${ }^{\circledR}$ (Keeler Ltd., Windsor, UK). The tear film classifications used are those proposed by Guillon in the 1990s, ${ }^{8-10}$ and those considered in this study are: open meshwork, close meshwork, wave, amorphous, interference fringe.

The division into two groups was made because at the end of each week every candidate had to complete a satisfaction questionnaire ${ }^{11}$ about symptoms related to CL use in order to monitor whether the non-carriers presented more symptoms. ${ }^{12,13}$ Each candidate was informed of the purpose and duration of the study. Before the first CL fitting, each subject signed a declaration of acceptance and was required to fill out the McMonnies questionaire $^{14,15}$ for early detection of dry eye symptoms. The symptoms were considered clinically significant only if they appeared after at least two days of lens use.

\section{Contact lenses}

Four different CL, three of different materials, were compared: Hioxifilcon, Omafilcon A and Filcon 1b, belonging respectively to Food and Drugs Administration (FDA) Groups 2, 2 and 4 (http://www.fda.gov/default.htm). The fourth lens, used as control and reference lens, was Etafilcon A, one of the first hydrogel materials approved by the FDA for disposable
Correspondence: Antonio Papagni, University of Milano-Bicocca, Department of Materials Sciences, via R. Cozzi 53, 20125, Milano, Italy. E-mail: antonio.papagni@unimib.it

Key words: soft contact lenses, pre-lens tear film analysis, evaluation scales, type of materials.

Received for publication: 30 September 2011. Revision received: 16 June 2012.

Accepted for publication: 28 August 2012.

This work is licensed under a Creative Commons Attribution NonCommercial 3.0 License (CC BYNC 3.0).

(C)Copyright A. Papagni et al., 2012

Licensee PAGEPress, Italy

Optometry Reports 2012; 2:e7

doi:10.4081/optometry.2012.e7

lenses. The three lenses under investigation are denoted with letter $\mathrm{T}$ followed by the numbers 1, 2 and 3; $\mathrm{C}$ indicates control lens. The physical properties and characteristics of the various lenses are shown in Table 1.

\section{Lens T1}

The Hioxifilcon lens is a polar copolymer (non-ionic) belonging to the family of disposable lenses, indicated for monthly replacement. The lens has a high molecular weight of 2-hydroxyethyl methacrylate (2-HEMA) and 2,3-diidrossipropilmethacrylate (glycerol methacrylate), and the crosslinking agent is ethylene glycol methacrylate. The lens consists of $41 \%$ Hioxifilcon A and a $59 \%$ water content by weight when immersed in a buffered saline, sodium bicarbonate or sodium perborate. Available lenses are either transparent or slightly blue-tinted [copper ftalocianato (2) blue] to enhance visibility. ${ }^{16,17}$

\section{Lens T2}

The Omafilcon A is a poly-HEMA modified with phosphorylcholine, which is the main monomer 2-methacryloyloxyethylphosphorylcoline. The curing agent is ethyleneglycol dimethacrylate. This lens is slightly blue because it is colored with Reactive Blue Dye N. 4 for easier handling. The lens is prescribed for daily use for the correction of refractive errors, including simple myopia and hyperopia, or with astigmatism up to $0.75 \mathrm{D}$. The lens is prescribed to be used for a month and is approved for correction of aphakia. ${ }^{18-21}$ The Omafilcon A lens has been used in numerous comparative studies because of its greater tolerance profile in patients with dry eye. ${ }^{22-24}$ A 1999 in vitro study ${ }^{25}$ reported that Omafilcon A lenses show a lower dehydration rate compared to other lenses containing HEMA+MA, HEMA and polyvinyl alcohol. 


\section{Lens T3}

The T3 lens is composed of two polymers: Filcon 1b, a synthetic polymer, and hyaluronic acid, a natural polymer. The hyaluronic acid provides enhanced hydration, more continuous lubrication, and stabilization of the tear film, thus improving subjective comfort. These enhanced lens characteristics allow for a weekly replacement-wearing schedule. Until now, only studies on customer satisfaction have been reported in the literature and no comparison with other lenses has so far been described. The hyaluronic acid is already employed as a mean component of artificial tears. The physical properties of the lens as provided by the manufacturer are listed in Table $2 .{ }^{26}$

\section{Control lens (C)}

The control lens is a hydrophilic soft lens with spherical geometry, comprising a copolymer of 2-HEMA and methacrylic acid. The cross-linking agents are 1,1,1-trimethylol propane trimethylolpropane trimethacrylate and ethylene glycol dimethacrylate. At hydrated state, the lens consists of a $42 \%$ HEMA+MA copolymer and a 58\% water content by weight when immersed in a buffered saline solution with sodium bicarbonate or sodium perborate. The lens is slightly tinted with Reactive Blue Dye N. 4 and Reactive Yellow Dye N. 86. The formulation also contains benzothiazole, a monomer with UV absorbing ability. The lens is suitable for daily use to be replaced every two weeks. ${ }^{27-29}$

\section{Study schedule}

The research was carried out over a 3-week study period. Every week, each candidate was fitted with a CL in the eye to be tested, while in the contralateral eye a traditional hydrogel lens (Etafilcon A) was fitted as control. The study was performed double blind; the patient did not know which of the two eyes had been fitted with the lens to be tested to ensure that response to the to the questionnaire administered at the end of each week was purely subjective. A clinical classification was made of the pre-lens tear film by evaluating the appearance of the front surface of the lens with Tearscope Plus ${ }^{\circledR}$ (Keeler Ltd.) at two specific time points: immediately after blinking, and $5 \mathrm{~s}$ after blinking. This classification is based on an in-house photographic scale reference produced by the authors. A digital slit lamp was used to capture the images for later evaluation.

Patients were examined after one week of daily wear. Patients were not told for how many hours the lenses could be worn. Before being examined, the patients had to have worn the lenses for at least $2 \mathrm{~h}$. Several images of the pre-lens tear film were collected in order to underline the differences in pre-lens tear film structure. Images were collected during two different photographic sessions. The first session of images was collected immediately after the subject had opened his eyes after blinking; that is when the tear film is well distributed on the anterior surface of the lens, forming the pre-lens tear film ${ }^{30,31}$ (time $\mathrm{t} 0$ ). The second set of images was collected $5 \mathrm{~s}$ after opening the eyelids after blinking (time t5). This time was chosen because, according to some research, blinking occurs every 5-6 s. ${ }^{32}$ In order to have a more stable tear film we took the series of photographs in the morning since it has been reported that the tear film is more stable at that time of day. ${ }^{33}$ In addition, another study has shown that the use of CL produces changes in the blinking rate, resulting in less frequent and incomplete lid closure. ${ }^{34,35}$
Photographic grading scale for the pre-lens tear film: photographic scale of dehydration

To classify the images, we used a photographic scale divided into five categories according to the different aspects of the prelens tear film: condition 1 corresponds to a well-structured pre-lens tear film, condition 5 corresponds to an unstable pre-lens tear film, with a clear evaporation of the aqueous phase. $^{36,37}$

The second series of images analyzes the light reflected from the front surface of the CL with the aim of assessing the optical quality of the tear film on the front surface of the CL. This is accomplished by introducing a semitransparent grid inside the cone of light in the Tearscope Plus ${ }^{\circledR}$ (Keeler Ltd.) and projecting its image on the pre-lens tear film. A specific grading scale has been developed to classify the images. This scale takes into account the progressive deformation of the reflected grid image on the lens as a result of evaporation of the aqueous component of the pre-lens tear film, and provides information concerning alterations of the tear film due to CL wear as suggested by Efron. ${ }^{38,39}$ The scale is divided into five levels numbered 1 to 5 . Grade 5 corresponds to the film pre-lens in a fully altered state while grade 1 indicates an intact, stable and uniform pre-lens tear film. This methodology allows us to evaluate the grade of grid deformation with the pre-lens tear film stability ${ }^{40}$ In addition to the photographic analysis, we also administered a weekly questionnaire to each CL wearer in order to investigate the number of hours of use, obtain information on subjective comfort, and to report any symptoms. We have taken special care to note which of the two lenses caused the sensation of dryness since the main cause of discomfort

Table 1. Properties of the contact lenses used in this study.

\begin{tabular}{|c|c|c|c|c|}
\hline Lens & T1 & $\mathrm{T} 2$ & T3 & C \\
\hline Material & $\begin{array}{l}\text { Hioxifilcon A } \\
\text { (HEMA+GMA) }\end{array}$ & $\begin{array}{l}\text { Omafilcon A } \\
(\mathrm{HEMA+PC})\end{array}$ & Filcon $1 \mathrm{~b}+$ hyaluronic acid & $\begin{array}{l}\text { Etafilcon A } \\
(\text { HEMA+MA) }\end{array}$ \\
\hline Refractive index & 1.404 (hydrated) & 1.38 (hydrated) & 1.42 (hydrated) & 1.39 (hydrated) \\
\hline Water content & $59 \%$ & $62 \%$ & $57 \%$ & $58 \%$ \\
\hline Oxygen permeability & $\begin{array}{l}18 \times 10^{-11} \text { Fatt's unit }\left(\mathrm{cm}^{2} / \mathrm{s}\right) \mathrm{x} \\
\left(\mathrm{mL} \mathrm{O}_{2} / \mathrm{mL} \times \mathrm{mm} \mathrm{Hg} \text { at } 35^{\circ} \mathrm{C}\right) \\
\text { Revised Fatt's method }\end{array}$ & $\begin{array}{l}27 \times 10^{-11} \text { Fatt's unit }\left(\mathrm{cm}^{2} / \mathrm{s}\right) \mathrm{x} \\
\left(\mathrm{mL} \mathrm{O} \mathrm{O}_{2} / \mathrm{mL} \times \mathrm{mm} \mathrm{Hg} \text { at } 35^{\circ} \mathrm{C}\right) \\
\text { Revised Fatt's method }\end{array}$ & $\begin{array}{l}28 \times 10^{-11} \text { Fatt's unit }\left(\mathrm{cm}^{2} / \mathrm{s}\right) \mathrm{x} \\
\left(\mathrm{mL} \mathrm{O} \mathrm{O}_{2} / \mathrm{mL} \times \mathrm{mm} \mathrm{Hg} \text { at } 35^{\circ} \mathrm{C}\right) \\
\text { Revised Fatt's method }\end{array}$ & $\begin{array}{l}20.2 \times 10^{-11} \text { Fatt's unit }\left(\mathrm{cm}^{2} / \mathrm{s}\right) \times \\
\left(\mathrm{mL} \mathrm{O}_{2} / \mathrm{mL} \times \mathrm{mm} \mathrm{Hg} \text { at } 35^{\circ} \mathrm{C}\right) \\
\text { Revised Fatt's method }\end{array}$ \\
\hline Central thickness & $0.14 \mathrm{~mm}$ & $0.065 \mathrm{~mm}$ & $0.06 \mathrm{~mm}$ & $0.07 \mathrm{~mm}$ \\
\hline Diameter & 14.2 & 14.2 & 14.0 & 14.2 \\
\hline FDA group & 2 & 2 & 4 & 4 \\
\hline Production method & Moulding & Moulding & Moulding & Moulding \\
\hline
\end{tabular}

The three lenses under investigation are denoted with letter T followed by the numbers 1, 2 and 3; C indicates control lens. HEMA, hydroxyethyl methacrylate; GMA, glycerol methacrylate; PC, phosphorylcholine; MA, methacrylic acid. 
reported by soft CL wearers is dry eye. ${ }^{41,42}$

We devised a scale using images collected with a slit lamp biomicroscope equipped with Tearscope Plus ${ }^{\circledR}$ (Keeler Ltd.) using a new grading scale. Classical Tearscope ${ }^{\circledR}$ (Keeler Ltd.) classification has 5 degrees. An optimal pre-lens lipid layer (thickness $>2 \mu \mathrm{m}$ ), is not colored but it will appear gray with reddish fringes as its quality decreases. If its thickness falls below $2 \mu \mathrm{m}$, reddish fringes become more pronounced and dry spots can appear. The dry spots can be of a different shape and extension depending on the extent to which tear film stability has been reduced. ${ }^{9}$

The colors of the photographic dehydration scale are described below.

The first image (Figure 1, left) shows a stable, uniform and gray-colored pre-lens tear film (subsequently reported as pattern Q1). In this case, the pre-lens film preserves a good dynamic in the later stages of blinking.

Image pattern Q2 (Figure 1, right) shows a thin but still stable pre-lens tear film with no zones of discontinuity. Many fringes are visibly bluish or tending to green. A few moments later, after a further reduction in thickness, the first fringe of red may appear.

Image pattern Q3 (Figure 2, left) shows a hybrid condition in which the pre-lens film is thicker but there are changes in its structure. It is still gray or slightly colored in appearance but most important is the evidence of particles in the pre-corneal tear film that undermine its stability. The corpuscles may be due to deposits on the lens surface or external agents that have contaminated the structure of the tears (cosmetics, debris from lashes or other external sources). This is a very common finding in traditional hydrogel soft CL wearers, and was also observed during this study. In spite of this, the tear film still covers the entire surface of the lens and there are no apparent dry areas.

Pattern 4 (Figure 2, right) is a state of alteration of pre-lens film. It represents an example of where the surface of the lens reveals the first dry spot or stripe.

The condition showed in pattern Q5 (Figure 3) concerns a completely non-wettable lens surface. There are no longer spots or stripes but rather a condition of a clearly unstable surface. The lens in this case looks like orange peel, and also the optical quality of its surface is totally compromised. The dehydration photographic scale of the prelens tear film was created in two versions: for dark eyes and for light eyes, in order to achieve a better clinical evaluation.

In parallel, we have also developed another grading scale (photographic scale of dehydration with grid) to evaluate lens surface quality after blinking and pre-lens tear film evaporation over time. To achieve this, a thin grid has been applied to the cone of light
Table 2. Results of the frecuency of each pattern registered with each lens.

\begin{tabular}{lcccccc} 
Grid scale & \multicolumn{2}{c}{ Lens T1 } & \multicolumn{3}{c}{ Lens T2 } & Lens T3 \\
Condition 1 & 15 & 2 & 16 & 1 & 17 & 1 \\
Condition 2 & 14 & 2 & 18 & 11 & 11 & 4 \\
\hline Condition 3 & 12 & 16 & 5 & 10 & 14 & 18 \\
Condition 4 & 1 & 14 & 1 & 16 & 0 & 13 \\
\hline Condition 5 & 0 & 8 & 0 & 2 & 0 & 6 \\
Total eyes examined & 42 & 42 & 40 & 40 & 42 & 42 \\
\hline
\end{tabular}

The three lenses under investigation are denoted with letter T followed by the numbers 1, 2 and 3 .

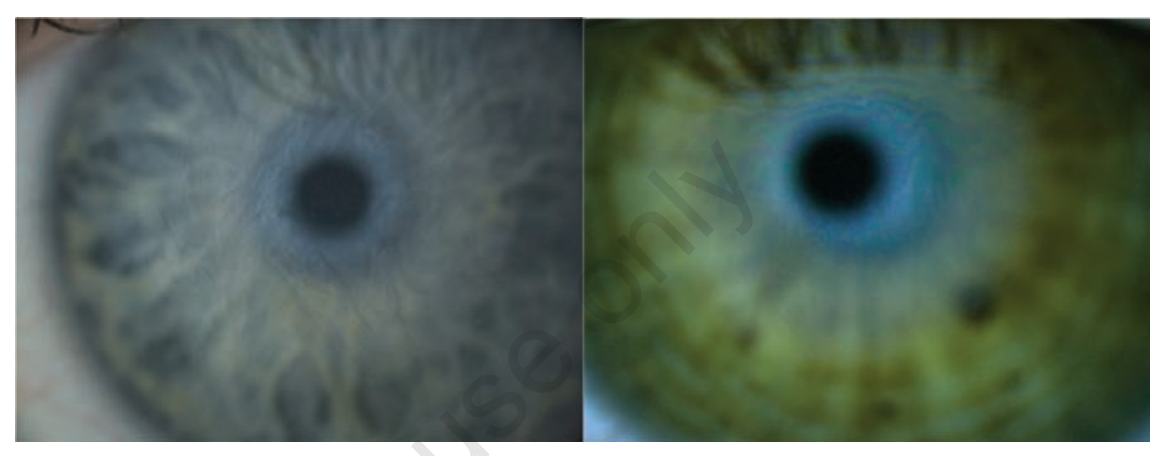

Figure 1. Dehydration scale pattern Q1 (left) and Q2 (right).

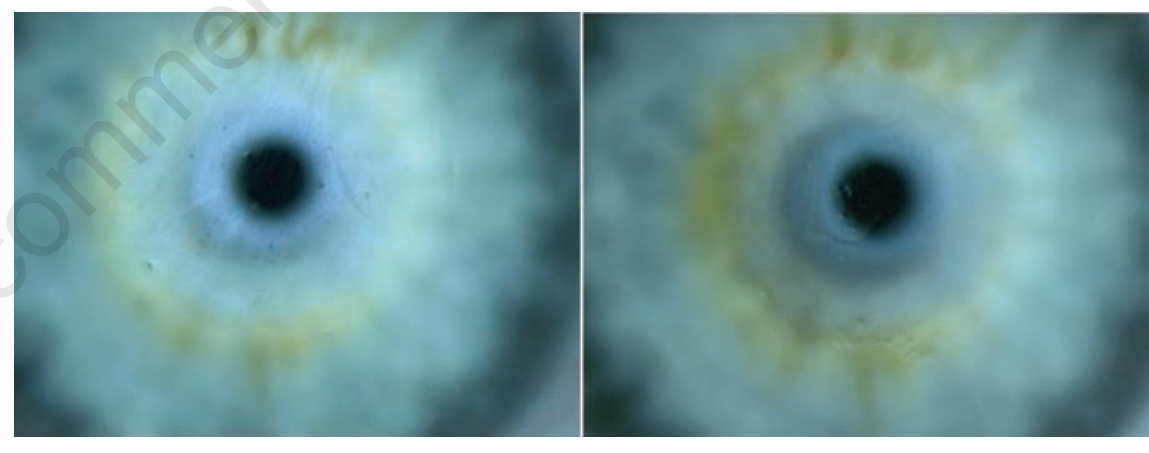

Figure 2. Dehydration scale patterns Q3 (left) and Q4 (right).

Tearscope ${ }^{\circledR}$ (Keeler Ltd.). This grid allows both break points of the film to be observed. These are seen as interruptions of the grid reflex on the surface of the pre-lens tear film, and small distortions of the grid reflex due to impurities on the surface of the lens (Figure 4). Indeed, a good optical quality of the surface is required for stable vision and for good contrast sensitivity. ${ }^{43-45}$

In Pattern 1 (Figure 4, left), the grating reflection on the surface of the lens is smooth and continuous, without any distortion or discontinuity of the grid reflex. In condition 2, the grid reflex shows initial discontinuity or

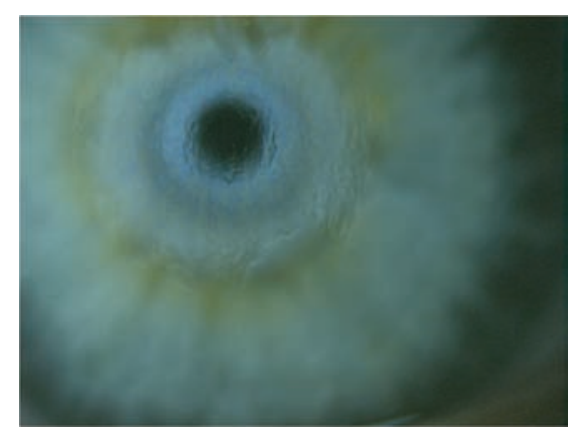

Figure 3. Dehydration scale pattern Q5. 
alteration. In condition 3 , the discontinuity of the grid reflex is clearly evident, but affects less than one-third of the front surface of the lens. In condition 4 (Figure 4, right), areas of discontinuity are more appreciable but do not exceed half of the lens surface. Finally, in condition 5 , the area of discontinuity is more than half of the lens surface. The grid reflex is, in some parts of the lens surface, no longer detectable, and this condition may be associated with symptoms of unstable or fluctuating vision.

This grid was produced in a single version because the color of the iris did not affect the evaluation of the pattern as the Tearscope ${ }^{\circledR}$ (Keeler Ltd.) with the grid inserted operates in low light conditions.

The assessment of dehydration of the CL was performed in vivo by means of the selfproduced photographic scale of dehydration (without the grid), and each lens was evaluated at the end of the week of daily wear use.

\section{Results}

Forty subjects completed the study wearing the three types of lenses under investigation in one eye and the control lens in the other one in a randomized study design.

\section{Dehydration measurements}

Table 2 shows the frequencies of the prelens tear film patterns [Tearscope ${ }^{\circledR}$ (Keeler Ltd.) with grid inserted] measured at time t0 s (at eye opening) and at time $t 5$ ( $5 \mathrm{~s}$ after the last blink). It is clear that at time $\mathrm{t} 0$, patterns represent a more stable pre-lens tear film while after $5 \mathrm{~s}$ degenerated patterns predominate.

Data reported in Table 2 show that with all CL, a non-optimal pre-lens tear film quality is observed in more than $50 \%$ of the wearers (conditions 2 and 3 ) even at $\mathrm{t} 0$. This is more evident at $\mathrm{t} 5$ and differences related to the type of material appear clearer. Indeed, approximately $25 \%$ of the CL wearers show a level 2 after $5 \mathrm{~s}$ while with the other lenses this condition is found in less than $10 \%$ of the wearers. When considering levels 3 and 4 together, again the T2 lens shows a better performance in terms of pre-lens tear film quality. Therefore, the change occurring between time 0 (eye opening) and time $\mathrm{t} 5$ is the most interesting finding and can be used to express the tear film variation time or in vivo dehydration speed of the CL surface. Expressing the tear film variation time by the symbol $\Delta$, the data collected in Table 2 can be represented graphically reporting the evaporation speed in function of type of materials.

The graph in Figure 5 shows the frequency of photographic pattern variation $(\Delta)$ found
Table 3. Reported symptoms per group of contact lenses wearers.

\begin{tabular}{|c|c|c|c|c|c|c|}
\hline \multirow[t]{2}{*}{ Symptom } & \multicolumn{3}{|c|}{ Group $1(W)$} & \multicolumn{3}{|c|}{ Group 2 (NF) } \\
\hline & $\mathrm{T} 1 \mathrm{~W}(\%)$ & $\mathrm{T} 2 \mathrm{~W}(\%)$ & T3W (\%) & T1NF (\%) & T2NF (\%) & T3NF (\%) \\
\hline Red eyes & 4.8 & 0.0 & 9.5 & 2.4 & 0.0 & 14.3 \\
\hline Dryness & 16.7 & 20.0 & 26.2 & 14.3 & 15.0 & 19.0 \\
\hline Feeling sand & 7.1 & 0.0 & 7.1 & 0.0 & 5.0 & 2.4 \\
\hline Itching & 4.8 & 7.5 & 2.4 & 9.5 & 10.0 & 11.9 \\
\hline Burning & 2.4 & 2.5 & 2.4 & 2.4 & 0.0 & 4.8 \\
\hline Foreign body sensation & n $\quad 11.9$ & 2.5 & 11.9 & 14.3 & 5.0 & 23.8 \\
\hline Tearing & 2.4 & 0.0 & 0.0 & 0.0 & 2.5 & 4.8 \\
\hline Photophobia & 4.8 & 2.5 & 4.8 & 2.4 & 0.0 & 0.0 \\
\hline Tired eyes & 11.9 & 5.0 & 9.5 & 9.5 & 2.5 & 11.9 \\
\hline Unstable vision & 2.4 & 5.0 & 7.1 & 4.8 & 2.5 & 7.1 \\
\hline General discomfort & 7.1 & 5.0 & 11.9 & 2.4 & 0.0 & 2.4 \\
\hline Burning at lens insertion & on 0.0 & 0.0 & 0.0 & 2.4 & 2.5 & 0.0 \\
\hline Irritation & 2.4 & 2.5 & 0.0 & 2.4 & 0.0 & 2.4 \\
\hline
\end{tabular}

NF, new fit; $W$, contact lens wearers.

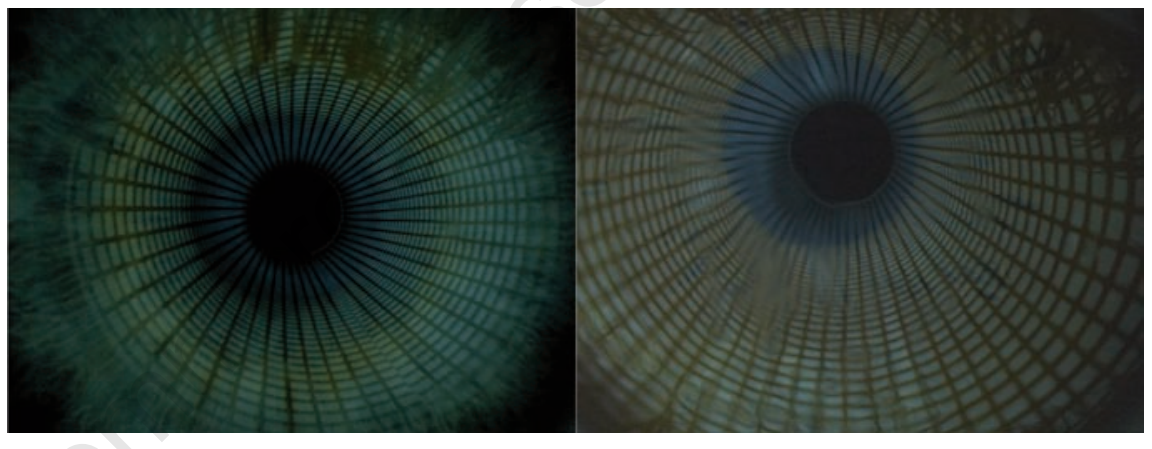

Figure 4. Optical quality dehydration scale pattern Q1 (left) and Q4 (right).

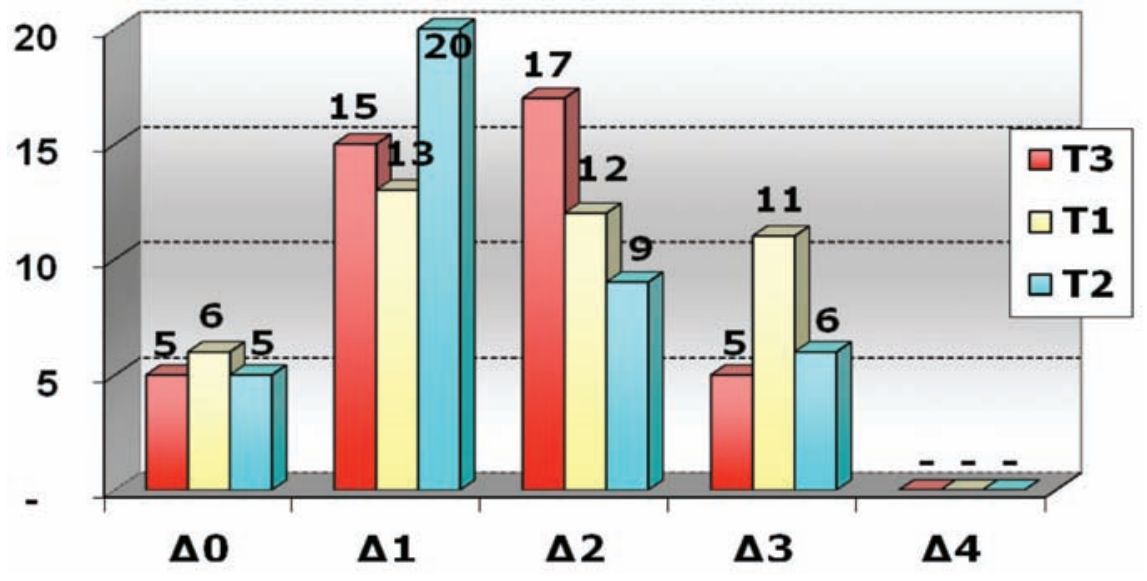

Figure 5. Frequency of pattern variations between t 0 and $\mathrm{t} 5$ in lenses $\mathrm{T} 1, \mathrm{~T} 2$ and $\mathrm{T} 3$. The $y$-axis reports the number of cases for each $\Delta$ value. The control lens $(C)$ with 4 cases of $\Delta 0$ variations. 
between time $0 \mathrm{~s}(\mathrm{t} 0)$ and time $5 \mathrm{~s}(\mathrm{t} 5)$.

The $\Delta 0$ indicates there is no variation in the photographic patterns between t0 (after blink) and 55 ( $5 \mathrm{~s}$ after blink) while $\Delta 1, \Delta 2$, $\Delta 3, \Delta 4$, respectively, represent variations of 2 , 3 or 4 degrees in the scale (i.e. $\Delta 2$ is recorded if $\mathrm{t} 0$ pattern is $\mathrm{Q} 1$ while $\mathrm{t} 5$ pattern is $\mathrm{Q} 3$ ).

The level of variation $\Delta 0$ remains almost constant for all lenses (6 T1, 5 T2, 5 T3, respectively); this factor may be due to the presence of a good tear film pattern, which does not lead to instability of the film prelens. The control lens (C), with 4 cases of $\Delta 0$ variations, confirms this.

$\Delta 1$ variations are very frequent for T2 lenses $(n=20)$. This kind of variation represents a slow time- dependent modification of the aspect of the tear film pattern after blinking.

Level $2(\Delta 2)$ dehydration speed is frequent for T3 lenses $(n=17)$. This appears at first to be an advantage compared to T1 lenses, which have many $\Delta 3$ variations $(n=11)$. However, we must consider that, while dehydrating more quickly, T1 lenses show a larger number of pattern 3 (Q3) soon after blinking which represent a more stable pre-lens tear film.

Given these findings, T3 lenses dehydrate more slowly, but this starts from an already altered condition and leads, as shown in Table 2 , to a greater number of $\mathrm{Q} 5$ patterns in which the lens surface is very dry and altered.

Comparing the change in pattern between the lenses studied (T1, T2 and T3) with the control leness, non-statistical differences (paired t-test, $\mathrm{P}>0.05$ ) were found in $\mathrm{T} 1$ and T3 leness. Only T2 showed statistically significant differences (paired t-test, $\mathrm{P}>0.01$ ), so pre-lens tear film patterns with $\mathrm{T} 2$ lenses show a statistical difference to the patterns observed with the control lenses (C). However, further research is necessary to evaluate these differences.

The behavior of the control lens (C) was analyzed separately since it was influenced by which of the test lenses was worn by the other eye. In this case, the $\Delta 1$ and $\Delta 3$ variations are also interesting. In fact, it was noted that there is a significant increase in $\Delta 1$ conditions in the first week of the test and a decrease in $\Delta 3$ conditions in the third week. The level of $\Delta 2$ variation is constant with the control lenses (C). From these data, it seems that $\mathrm{C}$ lenses dehydrate at different rates depending on which test lens (T) is used by the other eye.

This observation has led us to believe that the behavior of the $\mathrm{C}$ lens may be influenced by the behavior or symptoms induced by the test lens (T) worn in the other eye (Figure 6 , $\mathrm{C} 1, \mathrm{C} 2, \mathrm{C} 3$ signifies control lens (C) in one eye and test lens (T1, T2 and T3) in the other eye).

\section{Subjective assessment}

Figure 7 shows the subjective assessment reporting the symptom frequency related to the type of symptoms described by the wearers for the three test lenses used.

The most frequently reported signs and symptoms are: redness, dryness, itching, foreign body sensation, eyes feeling tired, and general discomfort.

Dryness in soft CL wearers is the most common symptom, as already reported by many researchers; therefore, a high prevalence of this symptom was expected and it was interesting to see which lens was able to reduce it. The T3 lens is definitely that which more often produces a feeling of dryness compared to the others, particularly in regular wearers compared to new wearers or new CL fits in this study. T1 and T2 lenses showed similar behavior. The lowest frequency of symptoms of dryness was observed in new fit wearers. This can be ascribed to the fact that they are less familiar with the concept of dryness. An itching sensation is more frequent in new wearers with $\mathrm{T} 1$ and $\mathrm{T} 2$ test lenses and the least in wearers of T3 lens. T3 lenses produce a more intense itching sensation in regular wearers.

A foreign body sensation is especially frequent with T3 lenses ( $\mathrm{n}=15$ among regular (Group 1) and new (Group 2) wearers vs 11 for $\mathrm{T} 1$ and 3 for $\mathrm{T} 2$; in the case of $\mathrm{T} 1$, the greater central thickness compared to $\mathrm{T} 2$ and T3 should be taken into account). T2 lenses,

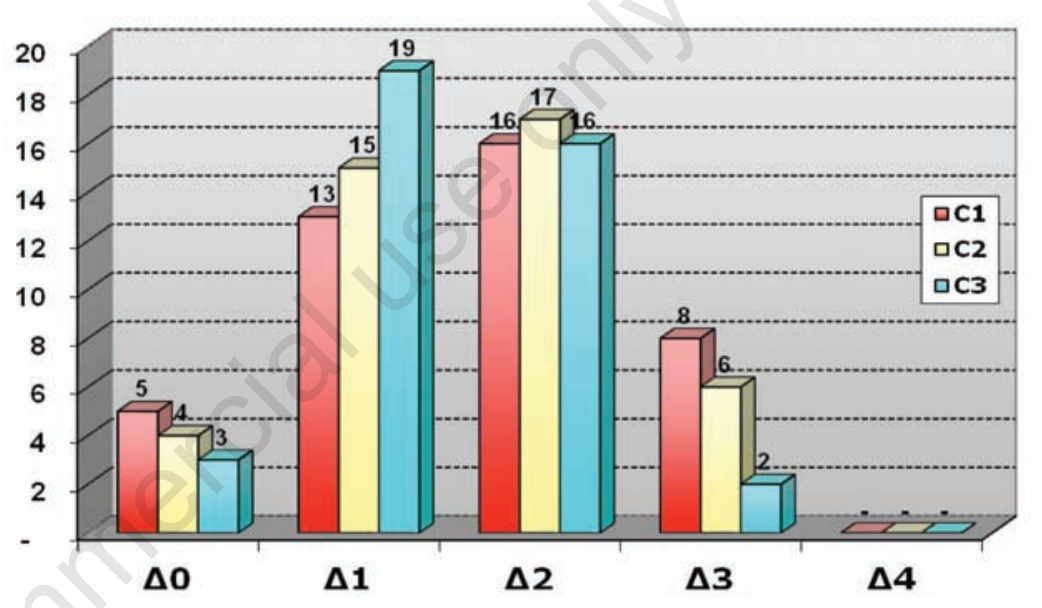

Figure 6. Dehydration changes in control lenses (C) during the 3-week study period, i.e. frequency of pattern variations between to and $t 5$ for the control lens $(C)$ over the three weeks. The $\mathrm{x}$-axis reports $\Delta$ and lens type worn in one eye (control lens in the other eye). The y-axis reports the number of cases for each $\Delta$ value.

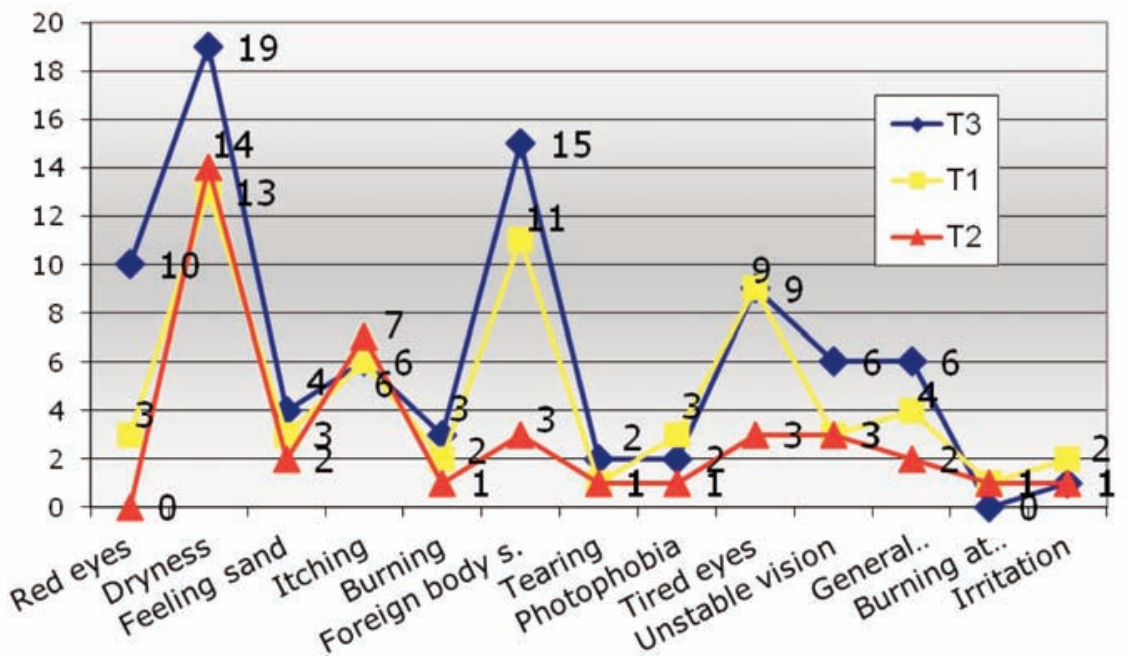

Figure 7. Frequency of reported symptoms. The y-axis reports the number of cases for each symptom ( $x$-axis). Yellow, T1 lenses; red, T2 lenses; blue, T3 lenses. 
having a comparable thickness and radius, show a notable lower foreign body frequency than T3 (Table 3). With respect to the general feeling of discomfort, T2 lenses give the best performance with 2 cases only in the regular wearers' group, followed by T1 with one case in the new wearers' group and 3 cases in the regular wearers' group. Also, in this case, T3 lenses showed the poorest performance with 6 cases in the new and in the regular wearers' groups. It should be noted that the symptoms observed with each test lens could be affected, to some extent, by the control lens (C) fitted in the other eye. This is an interesting effect that deserves to be investigated in more detail.

\section{Discussion}

A new grading scale has been developed to evaluate pre-lens tear film quality and stability, and to correlate them with the in vivo dehydration rate of the lenses worn. The grading scale has been applied to three different types of commercially available soft CL, allowing us to obtain valuable information regarding their in vivo behavior. Each lens investigated in this study has shown a different response towards the pre-lens tear film lens-drying rate. Results were influenced not only by the thickness and the composition of the pre-corneal tear film, but also by the type of materials constituting the lens itself.

All lenses tested here have been found to have a better tollerability profile with better hydrating patterns compared to traditional hydrogel lenses. In addition, changes in the behavior of the control lens (a traditional hydrogel) were compared to the test lenses. Indeed, it seems that the lens material affects to some extent the stability of the preceorneal tear film, especially the pre-lens tear film. After blinking, lenses T1, T3 and C behaved similarly; but there was a significant difference in the behavior of $\mathrm{T} 2$.

The pre-lens tear film is reduced and showed a greater dehydration rate in the control lens. Among the study lenses, T1 shows a faster dehydration, even though the dehydrated area is more limited than the other lenses which, in some cases, present wide surface breakup.

Differences in reported symptoms were recorded between the tested lenses. With the T2 lenses, new and regular wearers reported minor adverse symptoms. The T3 lenses appeared to be appreciated the least both in terms of adverse symptoms and overall comfort. Interestingly, the score assigned to control lenses (C) rose over the three weeks, depending on the score of the study lens fitted in the contralateral eye. We did not report any adverse ocular conditions with the control lenses during the test. All lenses presented good optical quality after blinking (t0) showing a good regular reflex. However, after $5 \mathrm{~s}$ (t5), all study lenses showed a better performance compared to traditional hydrogel. To summarize, a new grading scale to evaluate pre-lens tear film quality could be used to correlate the in vivo dehydration rate of the lenses worn with the patient symptomatology. However, further studies with more CL wearers and adequate statistical analysis is needed to explore their clinical utility in CL practice.

\section{References}

1. Rumpakis JMB. New data on contact lens dropouts: an international perspective. Rev Optom 2010;147:37-42.

2. Ramamoorthy P., Nichols J. Contact lens material characteristics associated with hydrogel lens dehydration. Ophthalmic Physiol 0pt 2010;30:160-6.

3. Maldonaldo-Codina C, Efron N. Dynamic wettability of p-HEMA-based hydrogel contact lens Ophthalmic Physiol Opt 2006;26:408-18.

4. Gonzalez-Meijome J. Dynamic in vitro dehydration patterns of unworn and worn silicone hydrogel contact lenses. J Biomed Mater Res B Appl Biomater 2009; 90:250-8.

5. Gonzalez-Meijome J. Qualitative and quantitative characterization of the in vitro dehydration process of hydrogel contact lenses. J Biomed Mater Res B Appl Biomater 2007;83:512-26.

6. Jones L, May C, Nazar L. In vitro evaluation of the dehydration characteristic of silicone hydrogel and conventional hydrogel contact lens materials. Cont Lens Anterior Eye 2002;25:147-56.

7. Begley CG, Caffery B. Use of the dry eye questionnaire to misure symptoms of ocular irritation in patients with acqueous tear deficient dry eye. Cornea 2002;21:664-70.

8. Guillon JP. The tear film structure of the contact lens wearers. PhD Thesis dissertation. London: The London City University; 1990.

9. Tearscope Plus manual. Windsor, UK: Keeler Limited; 1998.

10. Guillon JP. Non invasive Tearscope Plus routine for contact lens fitting. Cont Lens Anterior Eye 1998;21Suppl 1:S31-40.

11. Chalmers R, Begley C, Edrington T, et al. The agreement between self-assessment and clinician assessment of dry eye severity. Cornea 2005;24:804-10.

12. Begley C, Caffery B, Nichols K, Chalmers R. Responses of contact lens wearers to a dry eye survey. Optom Vis Sci 2000;77:406.

13. Guillon M, Maissa C. Dry eye symptomatology of soft contact lens wearers and non wearers. Optom Vis Sci 2005;82:82934.

14. McMonnies C, Ho A. Responses to a dry eye questionnaire from normal population. J Am Optom Assoc 1987;58:588-91.

15. McMonnies C, Ho A, Wakefield D. Optimum dry eye classification using questionnaire responses. Adv Exp Med Biol 1998;438:835-8.

16. Food and Drugs Administration. 510(k) Summary of safety and effectiveness K992692 (18 0ct 1999). Available from: http://www.accessdata.fda.gov/cdrh_docs/ pdf/K992692.pdf

17. Food and Drugs Administration. 510(k) Summary of safety and effectiveness K040303 (02 Feb 2005). Available from: http://www.accessdata.fda.gov/cdrh_docs/ pdf4/K040303.pdf

18. Food and Drugs Administration. 510(k) Summary of safety and effectiveness K974408 (16 Apr 1998). Available from: http://www.accessdata.fda.gov/cdrh_docs/ pdf/K974408.pdf

19. Food and Drugs Administration. 510(k) Summary of safety and effectiveness K032873 (11 Jul 2003). Available from: http://www.accessdata.fda.gov/cdrh_docs/ pdf3/K032873.pdf

20. Food and Drugs Administration. 510(k) Summary of safety and effectiveness K050717 (13 May 2005). Available from: http://www.accessdata.fda.gov/cdrh_docs/ pdf5/K050717.pdf

21. Food and Drugs Administration. 510(k) Summary of safety and effectiveness K970095/952152. Available from: http://www.accessdata.fda.gov/scripts/cdr $\mathrm{h} /$ devicesatfda/index.cfm?db=PMN\&id= K970095.

22. Lemp M. Report of the National Eye Institute/Industry Workshop on Clinical Trias in Dry Eyes. CLAO J 1995;21:221-32.

23. Young G, Bowers R, Hall B, Port M. Clinical comparison of Omafilcon A with four control materials. CLAO J 1997; 23:249-58.

24. Young G, Bowers R, Hall B, Port M. Six month clinical evaluation of biomimetic Hydrogel contact lens. CLAO J 1997; 23:226-36.

25. Hall B, Jones S, Young G, et al. The oneye dehydratation of proclear compatible lenses. CLAO J 1999;25:233-7.

26. Leonardi A, Decastello N. Monitoraggio clinico lente corneale denominata "SafeGel 7 Days". Padova: Università di Padova, Dipartimento di Neuroscienze, U.0. di Clinica oculistica; 2005.

27. Food and Drugs Administration. 510(k) 
Summary of safety and effectiveness K962804 (01 Oct 1996). Available from: http://www.accessdata.fda.gov/cdrh_docs/ pdf/K962804.pdf

28. Food and Drugs Administration. 510(k) Summary of safety and effectiveness K980634 (22 Apr 1998). Available from: http://www.accessdata.fda.gov/cdrh_docs/ pdf/K980634.pdf

29. Food and Drugs Administration. 510(k) Summary of safety and effectiveness K030167 (01 Apr 2003). Available from: http://www.accessdata.fda.gov/cdrh_docs/ pdf3/K030167.pdf

30. Bron AJ, Tiffany JM, Gouveia SM, et al. Functional aspect of the tear film lipid layer. Exp Eye Res 2004;78:347-60.

31. Guillon JP, Morris J, Hall B. Evaluation of the pre-lens tear film forming on three disposable contact lenses. Adv Exp Med Biol 2002;506:901-15.

32. Hill R. The quantitative blink. Int Contact Lens Clin 1984;11:366-8.

33. Lira M. Comparison of the tear film clini- cal parameters at two different times of the day. Clin Exp Optom 2011;94:557-62.

34 . Holly F. Tear film physiology and contact lens wear: contact lens tear film interaction. Am J Optom Physiol Opt 1981; 58:331-41.

35. Patel S, Henderson R, Bradley L, et al. Effect of visual display unit use on blink rate and tear stability. Optom Vis Sci 1991;68:888-92.

36. Mathers W. Evaporation from the ocular surface. Exp Eye Res 2004;78:389-94.

37. Craig J, Tomlinson A. Importance of the lipid layer in human tear film stability and evaporation. Optom Vis Sci 1997; 74:8-13.

38. Efron N. Pre soft lens tear film. In: Efron N. Contact lens conplications, 2nd Edition. Oxford: Butterworth Heinemann; 2004.

39. Kopf M, Yi F, Iskander D. Tear film surface quality with soft contact lenses using dynamic videokeratoscopy. J Optom 2008; 1:14-21.
40. Willcox M, Pearce D, Tan M, et al. Contact lens and tear film interactions. Exp Med Biol 2002;506:879-84.

41. Begley C, Caffery B, Nichols K, Chalmers $R$. Responses of contact lens wearers to a dry eye survey. Optom Vis Sci 2000;77:406.

42. Chalmers R, Begley C. Dryness symptoms among an unselected clinical population with and without contact lens. Cont Lens Anterior Eye 2006;26:25-30.

43. Thibos LN, Hong X, Bradley A, Begley CG. Deterioration of retinal image quality due to breakup of the corneal tear film. Invest Ophthalmol Vis Sci 1999;40:S544.2875.

44. Timberlake GT, Doane MG, Bertera JH. Short term, low contrast visual acuity reduction associated with in vivo contact lens drying. Optom Vis Sci 1992;69:75560.

45. Bailey MD, Walline JJ, Mitchell GL, Zadnick K. Visual acuity in contact lens wearers. Optom Vis Sci 2001;78:726-31. 